hep-th/0612309

BROWN-HET-1477

\title{
Four-Loop Cusp Anomalous Dimension From Obstructions
}

\author{
Freddy Cachazo, $1, *$ Marcus Spradlin, $2,+$ and Anastasia Volovich ${ }^{2,+}$ \\ ${ }^{1}$ Perimeter Institute for Theoretical Physics, \\ Waterloo, Ontario N2J 2W9, Canada \\ ${ }^{2}$ Brown University, Providence, Rhode Island 02912, USA
}

\begin{abstract}
We introduce a method for extracting the cusp anomalous dimension at $L$ loops from four-gluon amplitudes in $\mathcal{N}=4$ Yang-Mills without evaluating any integrals that depend on the kinematical invariants. We show that the anomalous dimension only receives contributions from the obstructions introduced in hep-th/0601031. We illustrate this method by extracting the two- and three-loop anomalous dimensions analytically and the four-loop one numerically. The four-loop result was recently guessed to be $f^{(4)}=-\left(4 \zeta_{2}^{3}+24 \zeta_{2} \zeta_{4}+50 \zeta_{6}-4(1+r) \zeta_{3}^{2}\right)$ with $r=-2$ using integrability and string theory arguments in hep-th/0610251. Simultaneously, $f^{(4)}$ was computed numerically in hep-th/0610248 from the four-loop amplitude obtaining, with best precision at the symmetric point $s=t, r=-2.028(36)$. Our computation is manifestly $s / t$ independent and improves the precision to $r=-2.00002(3)$, providing strong evidence in favor of the conjecture. The improvement is possible due to a large reduction in the number of contributing terms, as well as a reduction in the number of integration variables in each term.
\end{abstract}

\footnotetext{
*Electronic address: fcachazo@perimeterinstitute.ca

${ }^{\dagger}$ Electronic address: spradlin@het.brown.edu

${ }^{\ddagger}$ Electronic address: nastja@het.brown.edu
} 


\section{INTRODUCTION}

$\mathcal{N}=4$ super Yang-Mills (MSYM) is a very fascinating theory. Recently, important progress has been achieved in two different but connected aspects of the theory. On the one hand, insights from integrability have led to a proposal [1, 2] for all-loop quantities such as the cusp anomalous dimension $f(g)$, which is the coefficient of the logarithmic correction to the anomalous dimension of large spin twist-2 operators [3, 4, 5, 6]. On the other hand, in [7] the integrand of the planar four-loop four-gluon scattering amplitude was found, and the amplitude was evaluated analytically to order $1 / \epsilon^{4}$ and numerically to order $1 / \epsilon^{2}$ in the dimensional regularization parameter $\epsilon=2-D / 2$.

The connection between these two remarkable results is that the coefficient $f^{(L)}$ of $g^{2 L}$ in the perturbative expansion of $f(g)$ controls part of the IR singular behavior of the $L$ loop scattering amplitude [8, 9, 10]. Hence one can in principle extract that coefficient, and thereby determine $f(g)$ order by order, by calculating four-gluon scattering amplitudes. However, in practice this is a very hard task. One reason for this is that the IR singularity for an $L$-loop amplitude begins at order $1 / \epsilon^{2 L}$, while the contribution from $f(g)$ only appears in the $1 / \epsilon^{2}$ singularity. Therefore, the larger $L$, the higher one has to climb into the $\epsilon$ expansion before uncovering the desired contribution from $f(g)$.

One of the results of [2] is a formula for $f(g)$ whose expansion can be written in the form

$$
f(g)=4 g^{2}-4 \zeta_{2} g^{4}+\left(4 \zeta_{2}^{2}+12 \zeta_{4}\right) g^{6}-\left(4 \zeta_{2}^{3}+24 \zeta_{2} \zeta_{4}+50 \zeta_{6}-4(1+r) \zeta_{3}^{2}\right) g^{8}+\mathcal{O}\left(g^{10}\right)
$$

where $r$ was conjectured to have the value $r=-2$ and $g^{2}=g_{\mathrm{YM}}^{2} N /\left(8 \pi^{2}\right)$. This formula refines an earlier guess in [11] which had the same form but $r=0$. The non-zero value of $r$ is due to a nontrivial phase factor in the spin chain S-matrix. Remarkably, the value $r=-2$ at four loops follows from a simple conjecture for the all-loop phase factor also given in [2] which has been shown to agree well with the AdS/CFT correspondence [12, 13].

On the other hand, the numerical evaluation of the $1 / \epsilon^{2}$ coefficient of the four-loop amplitude was carried out in [7]. The numerical calculation is done at different kinematical points $x=t / s$ with results $(r, x)$ given by $(-2.028(36), 1),(-2.059(36), 2),(-2.062(45), 3)$, $(-2.074(104), 15)$, where the quantities in parentheses denote the uncertainty in the last digits.

An unfortunate complication inherent in this approach is that the $L$-loop cusp anomalous dimension is just a number, but the $1 / \epsilon^{2}$ coefficient of the $L$-loop scattering amplitude is, 
in general, a very complicated function of the kinematic invariant $x=t / s$. Isolating this single number is like picking a needle out of a haystack. For example, the evaluation of the four-loop amplitude using Mellin-Barnes representations [14, 15, 16, 17] (the current state of the art) expresses the $1 / \epsilon^{2}$ coefficient as a sum of over 50,000 $x$-dependent terms ${ }^{1}$. Each of these terms is a multiple integral which in general is evaluated numerically.

The fact that an appropriate combination of these terms conspires to add up to an $x$-independent number highlights an inefficiency in this calculational approach. This is manifest in the fact that the numerical precision obtained by [7] depends on the value of $x$ where the evaluation is performed, as noted above, even though the result of the calculation cannot depend on $x$. In terms of $r$, the precision reported by [7] varies from approximately $1.5 \%$ at $x=1$ to $5 \%$ at $x=15$.

In this paper we demonstrate that the cusp anomalous dimension may be calculated in a manifestly $x$-independent manner, and with a significantly smaller set of terms with reduced number of integrations variables. The starting point at four loops is the formula [8, 9, 10, 18]

$$
\begin{gathered}
M^{(4)}(x, \epsilon)=\frac{1}{4}\left(M^{(1)}(x, \epsilon)\right)^{4}-\left(M^{(1)}(x, \epsilon)\right)^{2} M^{(2)}(x, \epsilon)+M^{(1)}(x, \epsilon) M^{(3)}(x, \epsilon) \\
+\frac{1}{2}\left(M^{(2)}(x, \epsilon)\right)^{2}+\frac{1}{4} f^{(4)} M^{(1)}(x, 4 \epsilon)+\mathcal{O}(1 / \epsilon),
\end{gathered}
$$

which relates the four-loop amplitude to a polynomial in lower-loop amplitudes, together with a 'correction' proportional to $f^{(4)}$. Rather than calculating both sides of this equation as functions of $x$, and then reading off $f^{(4)}$, as was done in [7], we show that $f^{(4)}$ (and more generally $f(g)$ ) only receives contributions from the 'obstructions' discovered in [19]. Moreover, only the lowest order obstructions contribute. It turns out that obstructions can be systematically extracted from $x$-dependent integrals and the rest can be thrown away term-by-term, eliminating the need to evaluate all of them and then rely on non-trivial cancellations. We carry out the relevant calculation analytically for the one-, two- and three-loop amplitudes. At four loops we use Czakon's MB program [17] as a starting point and then implement the systematic extraction of obstructions. Quite nicely, it turns out that a large number of the integrals that come out can be reduced by simple applications of Barnes lemmas and corollaries. This process was also automated. After performing the

\footnotetext{
${ }^{1}$ Each "term" here refers to an integral of a single rational expression of $\Gamma$ functions and their derivatives.
} 
remaining integrations numerically, we obtain

$$
f^{(4)}=-117.1789(2), \quad \text { or } \quad r=-2.00002(3),
$$

which is consistent with the prediction $r=-2$ within the precision of about $0.001 \%$.

In fact this computation of the cusp anomalous dimension is a simple application of a more general analysis of the structure of multi-loop amplitudes we present in this paper. Instead of working with amplitudes as functions of $x$, we work with their Mellin transforms to a new variable $y$. In $y$ space, any amplitude can be uniquely expressed as a sum of two kinds of terms. The first kind are singularities, which take the form of $\delta$-functions or derivatives of $\delta$-functions at $y=0$. These singularities are the obstructions introduced in [19]; in $x$-space they correspond to polynomials in $\log ^{2} x$. The remaining part of the amplitude is smooth at $y=0$. We show that the obstructions in $L$-loop amplitudes must separately satisfy any polynomial relation of the form (2) and that furthermore the cusp anomalous dimension only receives contributions from the leading singularity $\delta(y)$ at order $1 / \epsilon^{2}$. To summarize:

Our prescription for computing the cusp anomalous dimension is to read off the numerical coefficient in front of $\delta(y) / \epsilon^{2}$ on both sides of the Mellin transform of equation (2).

This paper is organized as follows. In section 2 we review how the cusp anomalous dimension may be extracted from the infrared singularities of gluon scattering amplitudes. In section 3 we define obstructions in detail, discuss their most important properties, and explain how they may be used to calculate the cusp anomalous dimension. In section 4 we illustrate the method at the two- and three-loop level exhibiting the form of the obstructions explicitly. In section 5 we give some details on the computation of the four-loop cusp anomalous dimension from obstructions. In section 6 we summarize our conclusions and list some possible promising directions for future work. Finally in appendix A the reader familiar with Mellin-Barnes representations will find detailed instructions for using Czakon's MB program to extract the obstructions from an amplitude. 


\section{STRUCTURE OF THE $L$-LOOP FOUR-GLUON AMPLITUDE}

We let $A^{(L)}(\epsilon, s, t)$ denote the planar $L$-loop four-gluon amplitude in $\mathcal{N}=4$ super-YangMills theory, dimensionally regulated to $D=4-2 \epsilon$ dimensions. We further define

$$
M^{(L)}(x, \epsilon)=(s t)^{\epsilon L / 2} A^{(L)}(s, t, \epsilon) / A^{(0)}(s, t), \quad x=t / s .
$$

It is convenient to divide by the tree-level amplitude because supersymmetry determines the helicity structure of the $L$-loop amplitude to be the same as that of the tree-level amplitude; therefore the ratio is a function only of $\epsilon$ and the Mandelstam invariants. The prefactor $(s t)^{\epsilon L / 2}$ is convenient because the resulting quantity $M^{(L)}$ is a function of the ratio $x=t / s$ only, and satisfies the symmetry

$$
M^{(L)}(x, \epsilon)=M^{(L)}(1 / x, \epsilon)
$$

However we caution the reader that it is not standard in the literature to factor out $(s t)^{\epsilon L / 2}$ as we have done.

Expressions for $M^{(L)}(\epsilon, x)$ in terms of a basis of integrals are known for $L=1,2,3,4$ ([20], [21], [18], [7] respectively). For $L<4$ the basis is determined by the structure of the simplest unitarity cuts, i.e, double cuts. This analysis led to the "rung rule" which basically states that the $L$-loop amplitude can be constructed from the diagrams that give rise to the $(L-1)$ loop amplitude by adding one rung to all diagrams in such a way that no internal bubbles or triangles are produced [21]. For $L \geq 4$, the rung rule gives only the part of the amplitude that can be determined by double cuts. The four-loop amplitude is then special in the sense that it is the first order in which the rung rule is incomplete.

In a remarkable recent effort [7] the two diagrams missing in the four-loop amplitude were found. There it was noticed that these two diagrams, as well as the ones produced by the rung rule, satisfy some intriguing conformality properties [22, 23] that have been studied recently in [24]. This new insight, together with some generalized unitarity arguments developed in [25, 26, 27, 28], might lead to a systematic way of generating the diagrams at any order in perturbation theory. Very recently a proposal for the integrand of the $L=5$

loop amplitude was presented [29]. In Figure 1 we show the integrals contributing to the $L \leq 4$ loop amplitudes. 


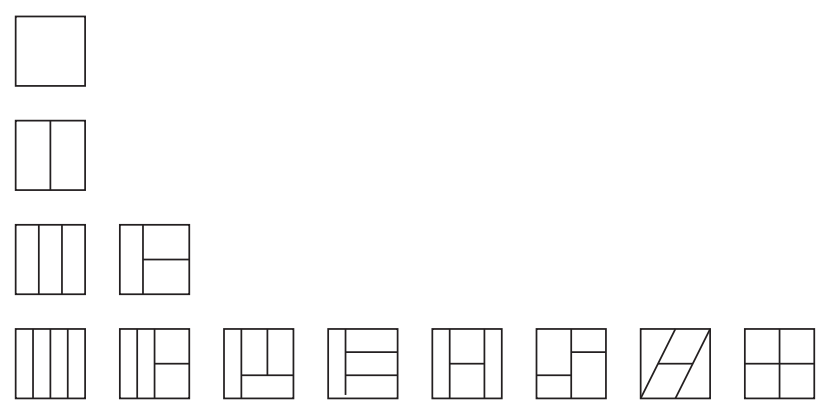

FIG. 1: Here we depict the topologies of the integrals which contribute to the $L$-loop four-gluon amplitude, for $L \leq 4$. The numerical coefficients and the numerator factors in the integrand are given by the rung rule for all but the last two four-loop diagrams. We refer the reader to [7] for further details.

\section{A. Infrared Singularity Structure}

Let us now review the known IR singularity structure of scattering amplitudes of gluons in $\mathcal{N}=4 \mathrm{SYM}$. The cusp anomalous dimension controls part of the $1 / \epsilon^{2}$ singularity and therefore it can be computed once the amplitudes are known explicitly.

In particular, it is known [8, 9, 10, 18] that the infrared singularities of an $L$-loop gluon amplitude, which contain $1 / \epsilon^{2 L}$ and lower poles, can be expressed as a polynomial in lowerloop amplitudes according to the formula

$$
M^{(L)}(x, \epsilon)=X^{(L)}\left[M^{(l)}(x, \epsilon)\right]+\frac{1}{4} f^{(L)} M^{(1)}(x, L \epsilon)+\mathcal{O}(1 / \epsilon)
$$

where the polynomials $X^{(L)}$ are conveniently encoded in the formula

$$
X^{(L)}\left[M^{(l)}(x, \epsilon)\right]=M^{(L)}(x, \epsilon)-\left.\log \left(1+\sum_{l=1}^{\infty} a^{l} M^{(l)}(x, \epsilon)\right)\right|_{a^{L} \text { term }}
$$

The one-loop amplitude takes the form

$$
M^{(1)}(x, L \epsilon)=-\frac{2}{L^{2} \epsilon^{2}}+\mathcal{O}(1)
$$

Therefore, (6) implies that the $L$-loop cusp anomalous dimension is given by the following deceivingly simple formula

$$
f^{(L)}=-2 L^{2}\left[M^{(L)}(x, \epsilon)-X^{(L)}\left[M^{(l)}(x, \epsilon)\right]\right]_{1 / \epsilon^{2}}
$$

where the subscript on the right instructs us to take the coefficient of the $1 / \epsilon^{2}$ singularity. 
Note that $f^{(L)}$ is a number; therefore the right hand side of (9) must be $x$ independent. The calculation of [7] does not make this $x$-independence manifest, so $f^{(4)}$ was computed for various values of $x$ as a consistency check, as mentioned in the introduction. The cancellation of the $x$ dependence in (9) is precisely the delicate step that we manage to avoid with our obstruction technique as we explain in the next section.

Relations similar to (6) hold in any massless gauge theory, but in $\mathcal{N}=4$ Yang-Mills theory it is believed that the stronger statement

$$
M^{(L)}(x, \epsilon)=X^{(L)}\left[M^{(l)}(x, \epsilon)\right]+\frac{1}{4} f^{(L)}(\epsilon) M^{(1)}(x, L \epsilon)+C^{(L)}+\mathcal{O}(\epsilon)
$$

holds, where $C^{(L)}$ are numerical constants and $f^{(L)}(\epsilon)$ are functions of $\epsilon$ only, with $f^{(L)}(0)=$ $f^{(L)}$ being the $L$-loop cusp anomalous dimension. This relation was originally proven for the two-loop four-particle amplitude in [30], and has been subsequently shown to hold for the three-loop four-particle amplitude [18] as well as the two-loop five-particle amplitude [31, 32].

Below we will make use of (10) at two and three loops, as well as (6) at four loops, so we record here the explicit forms of these relations,

$$
\begin{aligned}
M^{(2)}(x, \epsilon)= & \frac{1}{2}\left(M^{(1)}(x, \epsilon)\right)^{2}+\frac{1}{4} f^{(2)}(\epsilon) M^{(1)}(x, 2 \epsilon)+C^{(2)}+\mathcal{O}(\epsilon), \\
M^{(3)}(x, \epsilon)= & -\frac{1}{3}\left(M^{(1)}(x, \epsilon)\right)^{3}+M^{(1)}(\epsilon, x) M^{(2)}(x, \epsilon)+\frac{1}{4} f^{(3)}(\epsilon) M^{(1)}(x, 3 \epsilon)+C^{(3)}+\mathcal{O}(\epsilon), \\
M^{(4)}(x, \epsilon)= & \frac{1}{4}\left(M^{(1)}(x, \epsilon)\right)^{4}-\left(M^{(1)}(x, \epsilon)\right)^{2} M^{(2)}(x, \epsilon)+M^{(1)}(x, \epsilon) M^{(3)}(x, \epsilon) \\
& +\frac{1}{2}\left(M^{(2)}(x, \epsilon)\right)^{2}+\frac{1}{4} f^{(4)} M^{(1)}(x, 4 \epsilon)+\mathcal{O}(1 / \epsilon),
\end{aligned}
$$

where [18, 30]

$$
\begin{aligned}
\frac{1}{4} f^{(2)}(\epsilon) & =-\zeta_{2}-\zeta_{3} \epsilon-\zeta_{4} \epsilon^{2}+\mathcal{O}\left(\epsilon^{3}\right), \\
C^{(2)} & =-\frac{1}{2} \zeta_{2}^{2}, \\
\frac{1}{4} f^{(3)}(\epsilon) & =\frac{11}{2} \zeta_{4}+\epsilon\left(6 \zeta_{5}+5 \zeta_{2} \zeta_{3}\right)+\epsilon^{2}\left(c_{1} \zeta_{6}+c_{2} \zeta_{3}^{2}\right)+\mathcal{O}\left(\epsilon^{3}\right), \\
C^{(3)} & =\left(\frac{341}{216}+\frac{2}{9} c_{1}\right) \zeta_{6}+\left(-\frac{17}{9}+\frac{2}{9} c_{2}\right) \zeta_{3}^{2} .
\end{aligned}
$$

The quantities $c_{1}$ and $c_{2}$ are currently unknown, but they drop out of (10). The quantities $f^{(L)}(0)$ agree with the original calculations of the cusp anomalous dimension up to three loops carried out in [33, 34, 35]. 


\section{OBSTRUCTIONS AND THEIR APPLICATIONS}

In this section we first refine the definition of obstructions given in [19], clarifying their more formal mathematical structure. This allows us to give an all order in $\epsilon$ formula for the one-loop obstruction. We then explain the systematic way of implementing the extraction of obstructions at higher loops using as a starting point the program MB by Czakon [17]. Finally, we give the formula for the $L$-loop cusp anomalous dimension in terms of the obstructions at $\leq L$ loops.

\section{A. Obstructions-Theory}

The state-of-the-art technique for calculating $L$-loop integrals makes use of Mellin-Barnes representations [14, 15] (see [16] for a thorough introduction). This technique leads us to work not with an amplitude $M^{(L)}(x, \epsilon)$ but rather with its Mellin transform $F^{(L)}(y, \epsilon)$. These are related by

$$
M^{(L)}(x, \epsilon)=\int_{-i \infty}^{+i \infty} \frac{d y}{2 \pi i} x^{y} F^{(L)}(y, \epsilon) .
$$

It is fairly simple to write down an expression for $F^{(L)}(y, \epsilon)$ using the Feynman rules for any desired $L$-loop diagram. However these expressions are unwieldy because they typically involve multi-dimensional integrals of ratios of gamma functions. For example, the eight diagrams which contribute to the four-loop amplitude require Mellin-Barnes representations of dimensionalities from 10 to 14 .

In the representation (13) one typically cannot expand around $\epsilon=0$ under the integral sign because the function $F$ has singularities which collide to pinch the integration contour as $\epsilon \rightarrow 0$. We isolate these singularities using the formula

$$
\lim _{\omega \rightarrow 0} \frac{1}{y \pm \omega}=\mathcal{P} \frac{1}{y} \pm \pi \delta(y)
$$

and the generalization to higher singularities, given by taking derivatives of this equation with respect to $y$. The formula is missing some familiar factors of $i$ because the variable $y$ runs along the imaginary axis, and hence we take $\omega$ real $^{2}$.

\footnotetext{
${ }^{2}$ It is subtle to make sense of $\delta$-functions of complex arguments, and we make no attempt to do so here. Rather, we emphasize that in the analysis of this section we keep the $y$ contour precisely on the imaginary axis, meaning that one could rewrite all of the formulas in this section in terms of the real variable $z=-i y$ if one preferred. In this case one would have to take $\omega$ imaginary in (14)
} 
Isolating all of the singularities at $y=0$ leads to a formula of the form

$$
M^{(L)}(x, \epsilon)=f_{-i \infty}^{+i \infty} \frac{d y}{2 \pi i} x^{y} H^{(L)}(y, \epsilon)+P^{(L)}(\log x, \epsilon),
$$

where $f$ denotes a principal value integral, and we can now expand in $\epsilon$ under the integral sign. Since the Mellin transform of $\frac{\partial^{k}}{\partial y^{k}} \delta(y)$ is proportional to $\log ^{k} x$, the residual terms called $P^{(L)}$ in (15) are guaranteed to be polynomial in $\log x$ (order by order in $\epsilon$ ). These terms were called 'obstructions' in [19] because their presence indicates a failure to be able to assemble everything under a common $y$ integral at $\epsilon=0$. We see therefore that obstructions correspond to $\delta(y)$-function singularities in the Mellin transform of an amplitude. In fact, the symmetry (5) guarantees that $P^{(L)}(\log x, \epsilon)$ will be an even polynomial, and it also guarantees that $H^{(L)}(y, \epsilon)$ is an even function of $y$.

Let us illustrate this idea by calculating the obstruction to all orders in $\epsilon$ for the one-loop amplitude. This result is useful since the $1 / \epsilon^{2}$ term of $M^{(L)}(x, \epsilon)$ is controlled in part by the $\epsilon^{2 L-4}$ term of $M^{(1)}(x, \epsilon)$ as can be easily seen from (7).

In terms of the function

$$
F^{(1)}(y, \epsilon)=-\frac{1}{2} \frac{e^{\epsilon \gamma}}{\Gamma(-2 \epsilon)} \Gamma\left(1+\frac{1}{2} \epsilon+y\right) \Gamma^{2}\left(-\frac{1}{2} \epsilon+y\right) \Gamma^{2}\left(-\frac{1}{2} \epsilon-y\right) \Gamma\left(1+\frac{1}{2} \epsilon-y\right),
$$

the one-loop amplitude is given by

$$
M^{(1)}(x, \epsilon)=\int_{-i \infty}^{+i \infty} \frac{d y}{2 \pi i} x^{y} F^{(1)}(y, \epsilon), \quad-2<\epsilon<0 .
$$

As $\epsilon \rightarrow 0$, the two poles at $y= \pm \frac{1}{2} \epsilon$ collide with the integration contour. We can put those poles directly on top of the integration contour, with a principal value prescription, at the expense of subtracting off one-half of the appropriate residues as in (25). Therefore we find

$$
M^{(1)}(x, \epsilon)=f_{-i \infty}^{+i \infty} \frac{d y}{2 \pi i} x^{y} F^{(1)}(y, \epsilon)+\frac{1}{2} \operatorname{Res}_{y=+\epsilon / 2}\left[x^{y} F^{(1)}(y, \epsilon)\right]-\frac{1}{2} \operatorname{Res}_{y=-\epsilon / 2}\left[x^{y} F^{(1)}(y, \epsilon)\right] .
$$

The quantity $F^{(1)}(y, \epsilon)$ under the integral is to be expanded in $\epsilon$ inside the integral. The relative sign between the last two terms arises because the pole at $y=+\epsilon / 2$ approaches the integration contour from the left while the $y=-\epsilon / 2$ pole approaches the contour from the right. The obstruction is the sum of these two residues. Using the explicit formula (16), we find that the one-loop obstruction to all orders in $\epsilon$ is

$$
P^{(1)}(\log x, \epsilon)=\frac{\hat{c}_{\Gamma}(\epsilon)}{\epsilon}\left[\left(x^{+\epsilon / 2}+x^{-\epsilon / 2}\right)(\psi(1+\epsilon)-2 \psi(-\epsilon)+\psi(1))+\left(x^{+\epsilon / 2}-x^{-\epsilon / 2}\right) \log x\right],
$$


where $\psi(z)=\frac{d}{d z} \log \Gamma(z)$ and the prefactor contains the constant

$$
\hat{c}_{\Gamma}(\epsilon)=\frac{e^{\epsilon \gamma}}{2} \frac{\Gamma(1+\epsilon) \Gamma^{2}(1-\epsilon)}{\Gamma(1-2 \epsilon)} .
$$

As expected based on our general arguments, the $\epsilon$ expansion of the obstruction (19) is an even polynomial in $\log x$. We have also obtained an analytic formula for the two-loop obstruction to all orders in $\epsilon$, but the expression is quite lengthy and involves some remaining Mellin-Barnes type integrals that we do not know how to perform analytically at finite $\epsilon$.

\section{B. Obstructions-Implementation}

In the previous subsection we gave a formal definition of obstructions and used it to compute the one-loop obstruction to all orders in $\epsilon$. However, at higher loops the calculation rapidly becomes overwhelming, and must be implemented in a computer algebra system. Fortunately we can use Czakon's MB program which is designed to aid in the manipulation of Mellin-Barnes integrals as the starting point of the implementation. Let us now outline the steps we took in order to use MB to calculate obstructions. This outline will probably only be of interest to those with some familiarity with Mellin-Barnes integrals, or with MB in particular. A more concise outline of this procedure is given in the appendix.

We begin with a Mellin-Barnes representation for each integral $I$ contributing to the desired amplitude,

$$
I(x, \epsilon)=\int \frac{d z_{1}}{2 \pi i} \cdots \int \frac{d z_{n}}{2 \pi i} x^{f\left(z_{i}, \epsilon\right)} F\left(z_{i}, \epsilon\right),
$$

where $f\left(z_{i}, \epsilon\right)$ is a linear function of the $z_{i}$ and $\epsilon$, and $F\left(z_{i}, \epsilon\right)$ is in general a complicated ratio of $\Gamma$ functions whose arguments are linear in the $z_{i}$ and $\epsilon$ (a four-loop example is given below). The integration variables run from $-i \infty$ to $+i \infty$ along contours that can be chosen to be parallel to the imaginary axis, with appropriate real parts.

The representation (21) of $I(x, \epsilon)$ generically is not valid in a neighborhood of $\epsilon=0$, rather one has to start with a representation valid around some $\epsilon=\epsilon_{0}<0$. One then analytically continues the formula to a neighborhood of $\epsilon=0$, where one is free to develop the Laurent expansion of the amplitude that will exhibit all of the IR poles. In the process of this analytic continuation one must cross poles of the various $\Gamma$-functions in $F\left(z_{i}, \epsilon\right)$. The end result is the sum of the original integral, now valid in a neighborhood of $\epsilon=0$, plus a sum of residue terms which have fewer integration variables than the original integral. 
Once this process is completed, the coefficient at any desired order in $\epsilon$ is given as a sum of multiple integrals, most of them containing a factor of $x^{f\left(z_{i}\right)}$ to a power that is linear in the remaining integration variables. Some other integrals do not depend on $x$, or their $x$ dependence is an overall factor which is a power of $\log x$.

In each of the integrals which contains a factor of $x^{f\left(z_{i}\right)}$ we perform a linear change of variables $f\left(z_{i}\right) \rightarrow y$. The next step is to take all integrals that contain an $x^{y}$ factor and combine them under a single $y$ integral sign. Generically, this is impossible because the integration contour for $y$ can be different in each individual term. For example, two typical contributions might look like

$$
I_{1}(x)=\int_{\beta_{1}-i \infty}^{\beta_{1}+i \infty} \frac{d y}{2 \pi i} x^{y} \Gamma(y) H_{1}(y), \quad I_{2}(x)=\int_{\beta_{2}-i \infty}^{\beta_{2}+i \infty} \frac{d y}{2 \pi i} x^{y} \Gamma(y) H_{2}(y)
$$

where $\beta_{1}>0>\beta_{2}$ are on different sides of $y=0$. Therefore there is an obstruction at $y=0$ that prevents us from combining these two integrals. The obstruction is a simple pole in this case. Therefore, we can write

$$
I_{1}(x)+I_{2}(x)=\int_{\beta_{2}-i \infty}^{\beta_{2}+i \infty} \frac{d y}{2 \pi i} x^{y} \Gamma(y)\left(H_{1}(y)+H_{2}(y)\right)+\Delta
$$

where $\Delta$ is the obstruction, i.e, the contribution obtained from the residue at $y=0$,

$$
\Delta=\frac{1}{2 \pi i} \oint_{y=0} x^{y} \Gamma(y) H_{1}(y)
$$

As mentioned before, the presence of these obstructions that prevent one from naively combining all terms under the same integral sign were discovered in [19]. In that paper, the goal was to prove relations of the form (10) as an identity under the integral sign. Therefore, obstructions were undesired features of the Mellin-Barnes representation and differential operators were designed to annihilate them. Since our main goal in this paper is to compute the cusp anomalous dimension, it is precisely the obstructions that we are interested in.

In [19], we were content to collect all terms under a single with an arbitrary contour $\beta$. However, as we have already indicated in the previous subsection, it turns out that the most natural and useful notion is to take $\beta=0$ so that the $y$ contour sits along the imaginary axis, with a principal value prescription. If $\beta$ is infinitesimally small - which in practice means that it is sufficiently small to ensure that there are no more poles between the $y$ contour and 
the imaginary axis - then the final step of pushing the $y$ contour to the imaginary axis can be accomplished via

$$
\int_{\beta-i \infty}^{\beta+i \infty} \frac{d y}{2 \pi i} x^{y} H(y)=f_{-i \infty}^{+i \infty} \frac{d y}{2 \pi i} x^{y} H(y)+\frac{\operatorname{sign}(\beta)}{2} \operatorname{Res}_{y=0}\left[x^{y} H(y)\right]
$$

The final step in calculating the full obstruction in any amplitude $M(\epsilon, x)$ is to add up all of the contributing integrals $I(\epsilon, x)$. The above steps provide a constructive proof that any amplitude (and moreover, each individual integral $I(x, \epsilon)$ contributing to an amplitude) can be written in the form

$$
M(x, \epsilon)=f_{-i \infty}^{+i \infty} \frac{d y}{2 \pi i} x^{y} H(y, \epsilon)+P(\log x, \epsilon)
$$

for some $H(y, \epsilon)$ and $P(\log x, \epsilon)$. Note that the sum over contributing integrals $I(x, \epsilon)$ imposes that $M(\epsilon, x)$ satisfies the symmetry (5) . This ensures that the resulting $H(y, \epsilon)$, after all contributions are added together, satisfies $H(y, \epsilon)=H(-y, \epsilon)$, and that the obstruction $P(\log x, \epsilon)$ must be symmetric under $x \leftrightarrow 1 / x$, so it is an even polynomial in $\log x$.

\section{Cusp Anomalous Dimension From Obstructions}

Finally we explain how to exploit obstructions to simplify the calculation of the cusp anomalous dimension using equation (9). There are two essential properties of obstructions that enable this calculation.

The first essential property is that obstructions satisfy a product algebra-i.e. if $P_{1}$ and $P_{2}$ are the obstructions in two amplitudes $M_{1}$ and $M_{2}$, then the obstruction in the product $M_{1}(x, \epsilon) M_{2}(x, \epsilon)$ is simply the product of obstructions $P_{1}(\log x, \epsilon) P_{2}(\log x, \epsilon)$.

This is easy to see in $y$-space, where multiplication of $M_{i}(x, \epsilon)$ becomes convolution of the corresponding $F_{i}(y, \epsilon)$. Delta functions (or derivatives of delta functions) convolved with other delta functions still gives delta functions. However, delta functions convolved with a smooth function give a smooth function, as does of course a smooth function convolved with a smooth function.

This fact allows us to calculate, order by order, the obstructions in the 1,2,3, etc. loop amplitudes once and for all, and then they can subsequently be plugged into (9) to study the cusp anomalous dimension. Furthermore, any polynomial iteration relation of the form (10) that is satisfied by multi-loop amplitudes must also be satisfied by their corresponding 
obstructions. This is just the statement that we can take the Mellin transform of both sides of the equation and truncate to the terms proportional to (derivatives of) delta functions at $y=0$.

The second essential property is that the cusp anomalous dimension only receives contributions from obstructions, and in fact only from the leading obstruction proportional to $\delta(y)$ (as opposed to $\frac{\partial^{k}}{\partial y^{k}} \delta(y)$ for $k>0$ ). To prove this property, let us recall formula (9) for the $L$-loop cusp anomalous dimension

$$
f^{(L)}=-2 L^{2}\left[M^{(L)}(x, \epsilon)-X^{(L)}\left[M^{(l)}(x, \epsilon)\right]\right]_{1 / \epsilon^{2}}
$$

Use the decomposition (26) to write

$$
M^{(L)}(x, \epsilon)=\int_{-i \infty}^{+i \infty} \frac{d y}{2 \pi i} x^{y} \mathcal{P}\left[H^{(L)}(y, \epsilon)\right]+P^{(L)}(\log x, \epsilon)
$$

and

$$
X^{(L)}\left[M^{(l)}(x, \epsilon)\right]=\int_{-i \infty}^{+i \infty} \frac{d y}{2 \pi i} x^{y} \mathcal{P}\left[K^{(L)}(y, \epsilon)\right]+Q^{(L)}(\log x, \epsilon) .
$$

for some $K$ and $Q$. In fact the product algebra structure tells us that $Q^{(L)}(\log x, \epsilon)$ is given in terms of the obstructions in lower loop amplitudes by

$$
Q^{(L)}(\log x, \epsilon)=X^{(L)}\left[P^{(l)}(\log x, \epsilon)\right]
$$

Here we write the principal value integral as the integral of the principal part, with a conventional integral along the imaginary $y$-axis instead of $f$. The reason is that we would like to collect all of the terms in equation (27) under the single $y$ integral

$$
\begin{aligned}
\int_{-i \infty}^{+i \infty} \frac{d y}{2 \pi i} x^{y} \delta(y) f^{(L)}= & -2 L^{2} \int_{-i \infty}^{+i \infty} \frac{d y}{2 \pi i} x^{y} \mathcal{P}\left[H^{(L)}(y, \epsilon)-K^{(L)}(y, \epsilon)\right]_{1 / \epsilon^{2}} \\
& -2 L^{2} \int_{-i \infty}^{+i \infty} \frac{d y}{2 \pi i} x^{y}\left[P^{(L)}\left(\frac{\partial}{\partial y}, \epsilon\right)-Q^{(L)}\left(\frac{\partial}{\partial y}, \epsilon\right)\right]_{1 / \epsilon^{2}} \delta(y) .
\end{aligned}
$$

Note that we use $\delta$-functions that contain an extra normalization factor of $2 \pi i$ to compensate for our normalization of the $y$ integral. The $\epsilon$ expansion is performed inside the $y$ integral, as explained in the previous section, and the $1 / \epsilon^{2}$ coefficient of the term on the second line is a polynomial in $\partial / \partial y$ acting on $\delta(y)$ inside the integral.

Because equation (31) must hold for all $x$, the integrand must satisfy the equation pointwise in $y$. This means that the principal parts and the $\delta$-function parts must separately 
satisfy the equation, and in particular the coefficient of $\frac{\partial^{k}}{\partial y^{k}} \delta(y)$ must satisfy (31) separately for each $k$. By reading off the coefficient of $\delta(y)$ on both sides of (31) we conclude that

$$
f^{(L)}=-2 L^{2}\left[P^{(L)}(0, \epsilon)-Q^{(L)}(0, \epsilon)\right]_{1 / \epsilon^{2}} .
$$

Finally, using (30) we arrive at our main formula for computing the $L$-loop cusp anomalous dimension $f^{(L)}$ from the obstructions of $l \leq L$ loop amplitudes,

$$
f^{(L)}=-2 L^{2}\left[P^{(L)}(0, \epsilon)-X^{(L)}\left[P^{(l)}(0, \epsilon)\right]\right]_{1 / \epsilon^{2}}
$$

\section{ANALYTIC RESULTS AT ONE, TWO AND THREE LOOPS}

In this section we illustrate our method by showing that an analytic computation of $f^{(2)}$ and $f^{(3)}$ is possible without computing any $x$-dependent integrals. In order words, none of the technology available for evaluating Mellin-Barnes integrals in terms of harmonic polylogarithms is needed. We also show that relations like (10) that are expected to hold for the full amplitude must and do hold for the obstructions alone.

A careful analysis of the one-loop amplitude was carried out in section III.A and the formula (19) displays the obstructions in this amplitude to all orders in $\epsilon$. The computation of $f^{(L)}$ only requires the expansion of $P^{(1)}(\log x, \epsilon)$ through order $\epsilon^{2 L-4}$. Since in this paper we are interested in $L \leq 4$ it is sufficient to expand $P^{(1)}$ through order $\epsilon^{4}$ :

$$
\begin{aligned}
P^{(1)}(\log x, \epsilon)= & -\frac{2}{\epsilon^{2}}+\left[\frac{2 \pi^{2}}{3}+\frac{\log ^{2} x}{4}\right]+\epsilon\left[\frac{17 \zeta_{3}}{3}\right]+\epsilon^{2}\left[\frac{41 \pi^{4}}{720}+\frac{\pi^{2} \log ^{2} x}{24}+\frac{\log ^{4} x}{64}\right] \\
& +\epsilon^{3}\left[\frac{67 \zeta_{5}}{5}-\frac{59 \pi^{2} \zeta_{3}}{36}-\frac{11 \zeta_{3} \log ^{2} x}{24}\right] \\
& +\epsilon^{4}\left[-\frac{\pi^{6}}{4320}-\frac{70 \zeta_{3}^{2}}{9}-\frac{53 \pi^{4} \log ^{2} x}{5760}+\frac{\log ^{6} x}{4608}\right]+\mathcal{O}\left(\epsilon^{5}\right) .
\end{aligned}
$$

We have also obtained an exact formula for the obstructions in the two-loop amplitude. However instead of displaying the complete complicated formula we show its expansion through order $\epsilon^{2}$, which is sufficient for the purpose of isolating its contribution to $f^{(4)}$ :

$$
\begin{aligned}
P^{(2)}(\log x, \epsilon) & =\frac{2}{\epsilon^{4}}+\frac{1}{\epsilon^{2}}\left[-\frac{5 \pi^{2}}{4}-\frac{\log ^{2} x}{2}\right]+\frac{1}{\epsilon}\left[-\frac{65 \zeta_{3}}{6}\right] \\
+ & {\left[-\frac{\pi^{4}}{90}+\frac{\pi^{2} \log ^{2} x}{24}\right]+\epsilon\left[-\frac{463 \zeta_{5}}{10}+\frac{77 \pi^{2} \zeta_{3}}{12}+\frac{25 \zeta_{3} \log ^{2} x}{12}\right] } \\
+\epsilon^{2} & {\left[-\frac{1999 \pi^{6}}{30240}+\frac{95 \zeta_{3}^{2}}{18}+\frac{17 \pi^{4} \log ^{2} x}{720}+\frac{\pi^{2} \log ^{4} x}{32}+\frac{\log ^{6} x}{144}\right]+\mathcal{O}\left(\epsilon^{3}\right) . }
\end{aligned}
$$


At three loops we have worked out the obstructions through finite order, finding

$$
\begin{aligned}
P^{(3)}(\log x, \epsilon) & =-\frac{4}{3 \epsilon^{6}}+\frac{1}{\epsilon^{4}}\left[\frac{7 \pi^{2}}{6}+\frac{\log ^{2} x}{2}\right]+\frac{1}{\epsilon^{3}}\left[\frac{31 \zeta_{3}}{3}\right] \\
+ & \frac{1}{\epsilon^{2}}\left[-\frac{161 \pi^{4}}{3240}-\frac{7 \pi^{2} \log ^{2} x}{48}-\frac{\log ^{4} x}{32}\right] \\
+ & \frac{1}{\epsilon}\left[\frac{967 \zeta_{5}}{15}-\frac{965 \pi^{2} \zeta_{3}}{108}-\frac{25 \zeta_{3} \log ^{2} x}{8}\right] \\
+ & {\left[\frac{244261 \pi^{6}}{1632960}+\frac{107 \zeta_{3}^{2}}{18}-\frac{7 \pi^{4} \log ^{2} x}{576}-\frac{13 \pi^{2} \log ^{4} x}{256}-\frac{3 \log ^{6} x}{256}\right]+\mathcal{O}(\epsilon) . }
\end{aligned}
$$

It is a simple but delightful exercise to check that these three expressions obey the two- and three-loop iterative relations (10), (12). Although amusing, it is of course guaranteed from our general discussion of the properties of obstructions in the previous section.

\section{FOUR-LOOP CALCULATION}

In this section we explain the details of our numerical computation of the four-loop cusp anomalous dimension using obstructions.

The first step is to find an expression for the four-loop amplitude in terms of a small number of integrals. As mentioned in section II, the rung rule does not give the full amplitude for $L>3$. Finding the remaining contributions turns out to be a very hard problem. Luckily, in [7], the new non-rung-rule contributions were found and the full answer passed nontrivial consistency checks like unitarity and the correct IR singular behavior. Here we take the diagrammatic formula of [7], shown in Figure 1, as our starting point.

The second step is to find Mellin-Barnes representations for each of the integrals contributing to the amplitude. This is a straightforward process but such representations are not unique. Here we give as an illustration a 10-fold Mellin-Barnes representation for the four-loop ladder integral (the tenth integral $\int \frac{d y}{2 \pi i} x^{y}$ is suppressed)

$$
\begin{aligned}
F(y, \epsilon)= & \frac{e^{4 \epsilon \gamma}}{\Gamma(-2 \epsilon)} \int \prod_{i=1}^{9} \frac{d z_{i}}{2 \pi i} \Gamma(y-2 \epsilon) \Gamma(1+2 \epsilon-y) \Gamma\left(-1-\epsilon-z_{1}\right) \Gamma\left(-z_{1}\right) \Gamma\left(-z_{2}\right) \\
& \times \Gamma\left(1+z_{1}+z_{2}\right) \Gamma\left(-1-\epsilon-z_{3}\right) \Gamma\left(2+\epsilon+z_{1}+z_{3}\right) \Gamma\left(1-z_{2}+z_{3}\right) \\
& \times \Gamma\left(1-z_{1}-z_{2}+z_{4}\right) \Gamma\left(-2-2 \epsilon-z_{5}\right) \Gamma\left(z_{1}-z_{5}\right) \Gamma\left(-z_{4}+z_{5}\right) \\
& \times \Gamma\left(-3-2 \epsilon+z_{2}-z_{3}-z_{4}-z_{6}\right) \Gamma\left(-z_{6}\right) \Gamma\left(3+\epsilon+z_{3}+z_{5}+z_{6}\right) \\
& \times \Gamma\left(-3-2 \epsilon-y-z_{7}\right) \Gamma\left(1-z_{4}+z_{7}\right) \Gamma\left(-3-3 \epsilon-z_{8}\right) \Gamma\left(z_{5}-z_{8}\right) \\
& \times \Gamma\left(4+2 \epsilon+y+z_{8}\right) \Gamma\left(-z_{7}+z_{8}\right) \Gamma\left(-1-\epsilon+z_{4}+z_{6}-z_{7}-z_{9}\right)
\end{aligned}
$$




$$
\begin{aligned}
& \times \Gamma\left(-3-2 \epsilon-y-z_{8}-z_{9}\right) \Gamma\left(-z_{9}\right) \Gamma\left(3+\epsilon+y+z_{7}+z_{9}\right) \\
& \times \Gamma\left(1-z_{5}-z_{6}+z_{8}+z_{9}\right) / \\
& \left(\Gamma\left(-1-3 \epsilon-z_{1}\right) \Gamma\left(1-z_{2}\right) \Gamma\left(3+\epsilon+z_{1}+z_{3}\right) \Gamma\left(-2-4 \epsilon-z_{5}\right)\right. \\
& \left.\times \Gamma\left(1-z_{4}+z_{5}\right) \Gamma\left(1-z_{6}\right) \Gamma\left(-3-5 \epsilon-z_{8}\right) \Gamma\left(1-z_{7}+z_{8}\right) \Gamma\left(1-z_{9}\right)\right)
\end{aligned}
$$

The reason we display this one is that this representation has one less integration variable than the one given in [7], reflecting the non-uniqueness of Mellin-Barnes representations.

The third step is to extract the obstructions at order $1 / \epsilon^{2}$ from the Mellin-Barnes integrals. This step gives rise to a list with a large number of integrals. All these integrals are independent of $x$.

The fourth step is the simplification process. It turns out that a large fraction of these obstruction integrals can be reduced to integrals with a smaller number of integration variables by applications of Barnes lemmas and their corollaries (see appendix D of [16] for a nice collection of lemmas).

The result of this process is a formula for $f^{(4)}$ in terms of 135 -fold integrals, 1314 -folds and many more 3-, 2- and 1-folds. As an illustration we give the following "analytic" formula for the four-loop cusp anomalous dimension:

$$
\begin{aligned}
-\frac{1}{32} f^{(4)}=\left[\frac{1}{64} \int_{C_{5}} \prod_{i=1}^{5} \frac{d z_{i}}{2 \pi i} \frac{\Gamma\left(-z_{1}-z_{2}-2\right) \Gamma\left(-z_{2}\right) \Gamma\left(z_{1}-z_{3}+1\right) \Gamma\left(-z_{2}-z_{3}-2\right)}{\Gamma\left(-z_{2}-z_{3}-1\right) \Gamma\left(1-z_{4}\right) \Gamma\left(z_{1}+z_{4}+3\right)}\right. \\
\times \Gamma\left(-z_{3}\right) \Gamma\left(-z_{1}+z_{3}-z_{4}-1\right) \Gamma\left(-z_{4}\right) \Gamma\left(z_{1}+z_{4}+2\right) \Gamma\left(-z_{3}-z_{5}-1\right) \\
\times \\
\quad \times\left(-z_{3}-z_{4}-z_{5}-1\right) \Gamma\left(-z_{5}\right) \Gamma\left(z_{2}+z_{3}+z_{5}+2\right) \Gamma\left(z_{3}+z_{4}+z_{5}+1\right) \\
+\left[\frac{1}{12} \int_{C_{4}} \prod_{i=1}^{4} \frac{d z_{i}}{2 \pi i} \frac{\Gamma\left(-z_{1}-1\right) \Gamma\left(-z_{1}-z_{2}-2\right) \Gamma\left(-z_{2}\right) \Gamma\left(z_{1}+z_{2}+2\right) \Gamma\left(-z_{3}\right)}{\Gamma\left(-z_{1}-z_{2}-1\right)}\right. \\
\quad \times \Gamma\left(z_{2}+z_{3}+1\right) \Gamma\left(-z_{1}-z_{2}-z_{3}-z_{4}-1\right) \Gamma\left(-z_{4}\right) \Gamma\left(z_{1}+z_{4}+1\right) \\
\left.\quad \times \Gamma\left(z_{3}+z_{4}\right) \psi\left(-z_{1}-z_{4}-1\right) \psi\left(z_{3}+z_{4}+1\right)\right]+130 \text { similar terms } \\
+\left[\frac{5}{12} \int_{C_{3}} \prod_{i=1}^{3} \frac{d z_{i}}{2 \pi i} \frac{\Gamma\left(-z_{1}-2\right) \Gamma\left(z_{1}+2\right) \Gamma\left(-z_{2}-2\right) \Gamma\left(z_{1}-z_{2}\right) \Gamma\left(z_{2}+2\right)}{\Gamma\left(z_{1}+3\right)}\right. \\
\quad \times \Gamma\left(-z_{1}-z_{3}-1\right) \Gamma\left(-z_{2}-z_{3}-1\right) \Gamma\left(z_{2}+z_{3}+2\right) \Gamma\left(z_{1}+z_{2}+z_{3}+3\right) \\
\left.\quad \times \psi\left(-z_{1}-z_{3}\right)\right]+1305 \text { similar terms } \\
+\left[\frac{1}{16} \int_{C_{2}} \prod_{i=1}^{2} \frac{d z_{i}}{2 \pi i} \Gamma\left(-z_{1}\right)^{2} \Gamma\left(z_{1}\right) \Gamma\left(1-z_{2}\right) \Gamma\left(-z_{1}-z_{2}+1\right) \Gamma\left(z_{1}+z_{2}-1\right)\right. \\
\left.\times \Gamma\left(z_{1}+z_{2}\right) \psi\left(1-z_{2}\right)\right]+9227 \text { similar terms } \\
\times
\end{aligned}
$$




$$
\begin{aligned}
& +\left[-\frac{57661}{27648} \int_{C_{1}} \frac{d z_{1}}{2 \pi i} \Gamma\left(-z_{1}-1\right) \Gamma\left(-z_{1}\right) \Gamma\left(z_{1}+1\right)^{2} \psi^{\prime \prime \prime \prime}\left(z_{1}+2\right)\right] \\
& \text { +3719 similar terms } \\
& + \text { numbers. }
\end{aligned}
$$

In this expression the integration contours for each variable are straight lines parallel to the imaginary axis with real parts given respectively by

$$
\begin{aligned}
& C_{5}=\left[-\frac{6}{5},-\frac{24}{25},-\frac{9}{25},-\frac{8}{25},-\frac{4}{25}\right], \\
& C_{4}=\left[-\frac{17}{25},-\frac{12}{25},-\frac{4}{25},-\frac{3}{25}\right], \\
& C_{3}=\left[-\frac{7}{4},-\frac{5}{4},+\frac{1}{2}\right], \quad C_{2}=\left[-\frac{1}{4},+\frac{3}{4}\right], \quad C_{1}=\left[-\frac{1}{2}\right] .
\end{aligned}
$$

The final step is the numerical evaluation of these integrals, which we performed using CUBA's Cuhre algorithm [36] as implemented by Czakon's MB program. It is important to

mention that the main source of numerical error comes from 5 and 4 folds. The full list of integrals is available from the authors upon request.

In the simplification process we implemented only the first and second Barnes lemmas and some of their simplest corollaries. We believe that a more detailed analysis will show that some major simplifications are still possible and a fully analytic answer is within reach. We believe that this is a very important problem but we leave it for future work.

\section{CONCLUSIONS AND FUTURE DIRECTIONS}

In this paper we clarified the nature of 'obstructions' in $L$-loop four-gluon amplitudes in $\mathcal{N}=4$ Yang-Mills theory. Their name comes from the fact that they are the terms which obstruct writing an amplitude as an inverse Mellin transform of a smooth function near $\epsilon=0$. These obstructions were shown to possess all the information needed for the computation of the cusp anomalous dimension at $L$-loop order.

We implemented a systematic way of extracting obstructions, thus leading to a new method for computing the cusp anomalous dimension. As an application of our method we recovered the known two- and three-loop results already given in the literature [18, 30, 33, $34,[35]$.

We also used the recently obtained [7] integrand for the four-loop four-gluon amplitude, given by the diagrams shown in Figure 1, to compute numerically the four-loop cusp anoma- 
lous dimension. Our result is

$$
f^{(4)}=-117.1789(2)
$$

where the quantity in parentheses denotes the uncertainty in the last digit (as reported by the CUBA numerical integrator).

It would be highly desirable to find an analytic formula for $f^{(4)}$. This is something which is definitely within reach and we leave it for future work. However, we can try to see how far one can get with (40) and some additional assumptions. The first assumption one can make is the KLOV transcendentality principle [35, 37], which assigns a degree of trascendentality $k$ to $\zeta_{k}, 1$ to $\pi$ and zero to rational numbers. The degree of a monomial is computed as the sum of the degree of each factor. The transcendentality principle then states that $f^{(L)}$ is homogeneous of degree $2 L-2$. This means that $f^{(4)}$ must be a linear combination of $\pi^{6}, \zeta_{3}^{2}$, and $\pi^{3} \zeta_{3}$ with rational coefficients. Clearly, if we take this principle literally there is nothing we can conclude from (40) for there would be an infinite number of possibilities consistent with it.

Let us take a stronger form of the principle which was observed in integrability studies [11]. The idea is to allow only $\zeta_{k}$ numbers to appear in the monomials, and only with integer coefficients with sign $(-1)^{L+1}$. Then at four loops we would have the ansatz

$$
f^{(4)}=-\left(n_{1} \zeta_{2}^{3}+n_{2} \zeta_{2} \zeta_{4}+n_{3} \zeta_{6}+n_{4} \zeta_{3}^{2}\right)
$$

with four positive integers $n_{i}$. There is of course ambiguity in the choice of $\left(n_{1}, n_{2}, n_{4}\right)$ since $7 \zeta_{6}=4 \zeta_{2} \zeta_{4}$ and $5 \zeta_{2} \zeta_{4}=2 \zeta_{2}^{3}$. Then we can ask how many inequivalent choices $\left(n_{1}, n_{2}, n_{3}, n_{4}\right)$ are consistent with our result (40) within error bars. It turns out that there is only one choice, namely

$$
-\left(4 \zeta_{2}^{3}+24 \zeta_{2} \zeta_{4}+50 \zeta_{6}+4 \zeta_{3}^{2}\right) \approx-117.1788285
$$

which is the value conjectured in [2]. The next nearest value

$$
-\left(2 \zeta_{2}^{3}+\zeta_{2} \zeta_{4}+\zeta_{6}+73 \zeta_{3}^{2}\right) \approx-117.1801235
$$

is over six sigma away from our numerical data. For comparison, we note that even with the best precision obtained in [7] there are more than 200 inequivalent choices of $\left(n_{1}, n_{2}, n_{3}, n_{4}\right)$ within the error bars.

Another interesting problem for the future is to study the feasibility of a higher loop calculation. As suggested in [1], if the integrability result holds to all orders in perturbation 
theory, then there must be some hidden structure in field theory that allows the same power of computation. We hope that the theory of obstructions elaborated in this paper can be refined even more so as to produce a technique applicable in practice to all orders in perturbation theory. There are some indications to be hopeful about this. One is the fact that we were able to obtain a closed formula for the one loop obstruction to all orders in $\epsilon$ (see [38] for similar all-orders quantities). The second is that, assuming the transcendentality principle, the anomalous dimension should only receive contribution from the highest degree terms. The rest must cancel out. It turns out that in our calculation many integrals gave rise to terms of the "wrong" degree which later on cancel. It is conceivable that the terms of wrong degree might be isolated and thrown away systematically leaving a simple expression for the highest degree terms - mirroring the way in which our method of obstructions isolates and systematically throws away the $x$-dependent terms which we also know must cancel. We also leave this fascinating question for the future.

It is also very tempting to suggest that a five-loop calculation might be feasible with our technique or a further refinement of it. Something certain is that a five-loop computation of the anomalous dimension using the IR structure of the five-loop amplitude [29] and then directly using MB to numerically evaluate both sides of (2) is well beyond our current computational power (the numerical computation of the four-loop anomalous dimension presented here already required over a CPU-month on $3 \mathrm{GHz}$ Xeon computers).

It would be interesting to understand the connection between obstructions and the converse mapping techniques employed for example in [39] to find asymptotic expansions of amplitudes, and to see whether any of the simplifications we obtained can be applied to the direct calculation of the cusp anomalous dimension from three-point functions (as in [40]).

Finally it would be interesting to investigate the applicability of similar techniques to other theories, including possibly QCD.

\section{Acknowledgments}

MS and AV are grateful to A. Jevicki and D. Kosower for interesting related converations, to Z. Bern for several comments on the manuscript, and especially to I. Klebanov for encouragement to revisit the four-loop amplitude after the complete basis of integrals became

known [7]. The authors gratefully acknowledge the use of computer resources at the IAS 
School of Natural Sciences, where this project was started in the spring of 2006. The research of FC at the Perimeter Institute is supported in part by funds from NSERC of Canada and MEDT of Ontario. The research of MS is supported by NSF grant PHY-0638520. Any opinions, findings, and conclusions or recommendations expressed in this material are those of the authors and do not necessarily reflect the views of the National Science Foundation.

\section{APPENDIX A: IMPLEMENTATION DETAILS}

Here we provide a concise outline explaining a straightforward way to harness the power of Czakon's MB program to calculate the $L$-loop cusp anomalous dimension using the method of obstructions as outlined in the main text of this paper. Recall that to calculate the cusp anomalous dimension we first need to extract the numerical coefficient of $\delta(y) / \epsilon^{2}$ in the Mellin transform of the $L$-loop amplitude. This may be accomplished as follows:

1. For each diagram that contributes to the $L$-loop amplitude, find a valid Mellin-Barnes representation and run MB to read off the coefficient of the $1 / \epsilon^{2}$ pole. The result is a list $\mathrm{X}$ of $x$-dependent integrals valid at $\epsilon=0$. In each resulting term the $x$-dependence can take four possible forms: (a) independent of $x$, (b) proportional to a power of $\log x$, (c) $x$ to the power of a linear function of the remaining integration variables, or (d) as in (c) but with a prefactor of some power of $\log x$.

2. Throw away any integrals in $\mathrm{X}$ that contain any power of $\log x$ - these terms cannot contribute to the cusp anomalous dimension.

3. In the terms containing $x$ to some power, make a linear change of variables to a new variable $y$ to write the $x$ dependence in each term as $x^{y}$. Of course, the location of the $y$ contour will in general be different in each term.

4. Take each integral which contains $x^{y}$ and perform an analytic continuation (by running the MB program again) to shift the $y$ contour infinitesimally close to $y=0$. Here 'infinitesimally close' means, in practice, close enough to guarantee that there are no remaining poles between the $y$ contour and the imaginary $y$-axis. This procedure produces a new list of $x$-dependent integrals Y. The $x$-dependence in each term is either a power of $\log x, x^{y}$, or independent of $x$. 
5. Throw away any integrals in $\mathrm{Y}$ that contain any power of $\log x$.

6. Finally, for each term that still contains $\int d y x^{y}$, push the $y$ contour onto the imaginary axis using (25) and throw away the principal part as well as any terms containing $\log x$. The result is a list of $x$-independent integrals $\mathrm{Z}$.

7. Add together the integrals $\mathrm{Z}$ obtained from all of the diagrams contributing to the $L$ loop amplitude. The resulting set of integrals adds up to a number $s^{(L)}$. The interpretation of this number is that it is the coefficient of $\frac{1}{\epsilon^{2}} \delta(y)$ in the Mellin transform of the $L$ loop amplitude $M^{(L)}(x, \epsilon)$. For example, at four loops we find $s^{(4)} \approx-174.285193(6)$.

The $L$-loop cusp anomalous dimension is then given in terms of the number $s^{(L)}$ and the obstructions in the lower-loop amplitudes according to the formula (33)

$$
f^{(L)}=-2 L^{2}\left[s^{(L)}-X^{(L)}\left[P^{(l)}(0, \epsilon)\right]_{1 / \epsilon^{2}}\right]
$$

where $X^{(L)}$ is the polynomial defined in (6) above.

The full $L$-loop obstruction (including all of the $\log ^{k} x$ terms) can be computed without too much additional work. One simply follows the above outline, order by order in $\epsilon$, but without throwing away any $\log ^{k} x$ terms.

The above procedure could actually be simplified somewhat by making an appropriate change of variables to $y$ and choosing the contour for $y$ to lie along the imaginary axis in the original Mellin-Barnes representation, before running MB. One would then use MB to push $\epsilon$ infinitesimally close to $\epsilon=0$, and then finally use formula (25) for the final push to $\epsilon=0$, obtaining a principal value integral plus obstructions. Note that this is how we chose to present the calculation of (19). Although conceptually simpler, we did not employ this method at four loops because some experimentation revealed that this method seemed to give rise to a larger number of terms than the above procedure.

[1] N. Beisert, R. Hernandez and E. Lopez, "A crossing-symmetric phase for $\mathrm{AdS}_{5} \times S^{5}$ strings," JHEP 0611, 070 (2006) hep-th/0609044.

[2] N. Beisert, B. Eden and M. Staudacher, "Transcendentality and crossing," J. Stat. Mech. 0701, P021 (2007) hep-th/0610251. 
[3] G. P. Korchemsky, "Asymptotics of the Altarelli-Parisi-Lipatov Evolution Kernels of Parton Distributions," Mod. Phys. Lett. A 4, 1257 (1989).

[4] G. P. Korchemsky and G. Marchesini, "Structure function for large $x$ and renormalization of Wilson loop," Nucl. Phys. B 406, 225 (1993) hep-ph/9210281.

[5] S. S. Gubser, I. R. Klebanov and A. M. Polyakov, "A semi-classical limit of the gauge/string correspondence," Nucl. Phys. B 636, 99 (2002) hep-th/0204051.

[6] M. Kruczenski, "A note on twist two operators in $N=4$ SYM and Wilson loops in Minkowski signature," JHEP 0212, 024 (2002) hep-th/0210115.

[7] Z. Bern, M. Czakon, L. J. Dixon, D. A. Kosower and V. A. Smirnov, "The four-loop planar amplitude and cusp anomalous dimension in maximally supersymmetric Yang-Mills theory," hep-th/0610248,

[8] L. Magnea and G. Sterman, "Analytic continuation of the Sudakov form-factor in QCD," Phys. Rev. D 42 (1990) 4222.

[9] S. Catani, "The singular behaviour of QCD amplitudes at two-loop order," Phys. Lett. B 427, 161 (1998) hep-ph/9802439.

[10] G. Sterman and M. E. Tejeda-Yeomans, "Multi-loop amplitudes and resummation," Phys. Lett. B 552 (2003) 48 hep-ph/0210130.

[11] B. Eden and M. Staudacher, "Integrability and transcendentality," J. Stat. Mech. 0611, P014 (2006) hep-th/0603157.

[12] M. K. Benna, S. Benvenuti, I. R. Klebanov and A. Scardicchio, "A test of the AdS/CFT correspondence using high-spin operators," hep-th/0611135.

[13] J. Maldacena and I. Swanson, "Connecting giant magnons to the pp-wave: An interpolating limit of $\mathrm{AdS}_{5} \times S^{5}, "$ hep-th/0612079.

[14] V. A. Smirnov, "Analytical result for dimensionally regularized massless on-shell double box," Phys. Lett. B 460, 397 (1999) hep-ph/9905323.

[15] J. B. Tausk, "Non-planar massless two-loop Feynman diagrams with four on-shell legs," Phys. Lett. B 469, 225 (1999) hep-ph/9909506.

[16] V. A. Smirnov, "Evaluating Feynman Integrals," Springer tracts in modern physics, 211 (Springer, Berlin, Heidelberg, 2004).

[17] M. Czakon, "Automatized analytic continuation of Mellin-Barnes integrals," Comput. Phys. Commun. 175, 559 (2006) hep-ph/0511200. 
[18] Z. Bern, L. J. Dixon and V. A. Smirnov, "Iteration of planar amplitudes in maximally supersymmetric Yang-Mills theory at three loops and beyond," Phys. Rev. D 72, 085001 (2005) hep-th/0505205.

[19] F. Cachazo, M. Spradlin and A. Volovich, "Hidden beauty in multiloop amplitudes," JHEP 0607, 007 (2006) hep-th/0601031.

[20] M. B. Green, J. H. Schwarz and L. Brink, " $N=4$ Yang-Mills And $N=8$ Supergravity As Limits Of String Theories," Nucl. Phys. B 198, 474 (1982).

[21] Z. Bern, J. S. Rozowsky and B. Yan, "Two-loop four-gluon amplitudes in $N=4$ super-YangMills," Phys. Lett. B 401, 273 (1997) hep-ph/9702424.

[22] D. I. Kazakov, "The Method Of Uniqueness, A New Powerful Technique For Multiloop Calculations," Phys. Lett. B 133, 406 (1983).

[23] N. I. Usyukina and A. I. Davydychev, "Exact results for three and four point ladder diagrams with an arbitrary number of rungs," Phys. Lett. B 305, 136 (1993).

[24] J. M. Drummond, J. Henn, V. A. Smirnov and E. Sokatchev, "Magic identities for conformal four-point integrals," JHEP 0701, 064 (2007) hep-th/0607160.

[25] Z. Bern, L. J. Dixon, D. C. Dunbar and D. A. Kosower, "One loop n point gauge theory amplitudes, unitarity and collinear limits," Nucl. Phys. B 425, 217 (1994) hep-ph/9403226.

[26] Z. Bern, L. J. Dixon, D. C. Dunbar and D. A. Kosower, "Fusing gauge theory tree amplitudes into loop amplitudes," Nucl. Phys. B 435, 59 (1995) hep-ph/9409265.

[27] R. Britto, F. Cachazo and B. Feng, "Generalized unitarity and one-loop amplitudes in $N=4$ super-Yang-Mills," Nucl. Phys. B 725, 275 (2005) hep-th/0412103.

[28] E. I. Buchbinder and F. Cachazo, "Two-loop amplitudes of gluons and octa-cuts in $N=4$ super Yang-Mills," JHEP 0511, 036 (2005) hep-th/0506126.

[29] Talk at the UCLA workshop "Is $N=8$ supergravity finite?" by H. Johansson.

[30] C. Anastasiou, Z. Bern, L. J. Dixon and D. A. Kosower, "Planar amplitudes in maximally supersymmetric Yang-Mills theory," Phys. Rev. Lett. 91, 251602 (2003) hep-th/0309040.

[31] F. Cachazo, M. Spradlin and A. Volovich, "Iterative structure within the five-particle two-loop amplitude," Phys. Rev. D 74, 045020 (2006) hep-th/0602228.

[32] Z. Bern, M. Czakon, D. A. Kosower, R. Roiban and V. A. Smirnov, "Two-loop iteration of five-point $N=4$ super-Yang-Mills amplitudes," Phys. Rev. Lett. 97, 181601 (2006) hep-th/0604074. 
[33] S. Moch, J. A. M. Vermaseren and A. Vogt, "The three-loop splitting functions in QCD: The non-singlet case," Nucl. Phys. B 688 (2004) 101 hep-ph/0403192.

[34] A. Vogt, S. Moch and J. A. M. Vermaseren, "The three-loop splitting functions in QCD: The singlet case," Nucl. Phys. B 691 (2004) 129 hep-ph/0404111.

[35] A. V. Kotikov, L. N. Lipatov, A. I. Onishchenko and V. N. Velizhanin, "Three-loop universal anomalous dimension of the Wilson operators in $N=4$ SUSY Yang-Mills model," Phys. Lett. B 595, 521 (2004) [Erratum-ibid. B 632, 754 (2006)] hep-th/0404092.

[36] T. Hahn, "CUBA: A library for multidimensional numerical integration," Comput. Phys. Commun. 168, 78 (2005) hep-ph/0404043.

[37] A. V. Kotikov and L. N. Lipatov, "On the highest transcendentality in $N=4$ SUSY," hep-th/0611204.

[38] T. Gehrmann, T. Huber and D. Maitre, "Two-loop quark and gluon form factors in dimensional regularisation," Phys. Lett. B 622, 295 (2005) hep-ph/0507061.

[39] S. Friot, D. Greynat and E. De Rafael, "Asymptotics of Feynman diagrams and the MellinBarnes representation," Phys. Lett. B 628, 73 (2005) hep-ph/0505038.

[40] S. Moch, J. A. M. Vermaseren and A. Vogt, "The quark form factor at higher orders," JHEP 0508, 049 (2005) hep-ph/0507039. 